Nunes, CH \& Michaliszyn, MS (2020). Teaching methodologies and quality improvement in higher education. Research, Society and Development, 9(7): 1-19, e384973765.

\title{
Metodologias de ensino e melhoria da qualidade do ensino superior
}

Teaching methodologies and quality improvement in higher education Metodologías de enseñanza y mejora de la calidad de la enseñanza superior

Recebido: 18/04/2020 | Revisado: 02/05/2020 | Aceito: 09/05/2020 | Publicado: 18/05/2020

\section{Camila Henriques Nunes}

ORCID: https://orcid.org/0000-0002-1663-9445

Instituto Federal Fluminense, Brasil

E-mail: camila.nunes@iff.edu.br

Mario Sergio Michaliszyn

ORCID: https://orcid.org/0000-0003-3463-0257

Universidade Positivo, Brasil

E-mail: mario@up.edu.br

\section{Resumo}

As transformações sociais, econômicas e culturais trazem sempre como implicação a necessidade de mudanças no cenário educacional e, consequentemente, no processo de ensino-aprendizagem, sobretudo, quando estas se referem à formação profissional. A efetivação de mudanças requer adaptação de metodologias de ensino que proporcionem ao aluno compreensão, aplicação e aprimoramento do conhecimento. Nesse sentido, este artigo tem como objetivo realizar uma breve revisão sobre as metodologias de ensino, suas aplicações e experiências exitosas no âmbito do Ensino Superior. Trata-se de uma pesquisa de abordagem qualitativa, com revisão bibliográfica sobre a temática analisada. Observou-se que as experiências com tais metodologias ainda são bem pontuais, necessitando de maior difusão pelo currículo. Embora exitosas essas ainda aparecem de forma discreta na academia.

Palavras-chave: Ensino híbrido; Aprendizagem baseada em problemas; Sala de aula invertida.

\footnotetext{
Abstract

Social, economic and cultural transformations always imply the need for changes in the educational scenario and, consequently, in the teaching-learning process, especially when these refer to vocational training. The implementation of changes requires adaptation of
} 
teaching methodologies that provide the student with understanding, application and improvement of knowledge. In this sense, this article aims to make a brief review on teaching methodologies, their applications and successful experiences in Higher Education. It is a qualitative research, with a bibliographic review on the analyzed theme. It was observed that the experiences with such methodologies are still very punctual, requiring greater dissemination throughout the curriculum. Although successful, these still appear discreetly in the Higher Education.

Keywords: Hybrid teaching; Problem-based learning; Inverted classroom.

\section{Resumen}

Las transformaciones sociales, económicas y culturales envuelven la necesidad de cambios en el escenario educativo y, consecuentemente, en el proceso de enseñanza-aprendizaje, sobre todo, cuando estas se refieren a la formación profesional. La efectividad de cambios requiere la adaptación de metodologías de enseñanza que proporcionen al alumno comprensión, aplicación y perfeccionamiento del conocimiento. Bajo este aspecto este artículo tiene como objetivo realizar una breve revisión sobre las metodologías de enseñanza, sus aplicaciones y experiencias exitosas en el ámbito de la Enseñanza Superior. Es una investigación cualitativa, con una revisión bibliográfica sobre el tema analizado. Se observó que las experiencias con tales metodologías siguen siendo muy puntuales y requieren una mayor difusión en todo el plan de estudios. Aunque exitosas, estas aparecen discretamente en la Enseñanza Superior.

Palabras clave: Enseñanza híbrida; Aprendizaje basado en problemas; Sala de clase invertida.

\section{Introdução}

As mudanças econômicas, sociais, culturais e o franco processo de globalização e informação, característicos de uma emergente "sociedade do conhecimento", tornam necessários os debates e aprofundamento de estudos e pesquisas no campo da educação e, mais especificamente, do ensino superior.

A partir das mudanças ocorrem novas demandas no processo formativo dos futuros profissionais, que precisam estar qualificados e com capacidade de resolução de situações cada vez mais complexas. $\mathrm{O}$ ensino meramente tecnicista e as metodologias tradicionais de ensino-aprendizagem não suprem mais estas demandas.

O estudo das metodologias de ensino na educação superior possibilita o aporte teórico 
necessário para despertar o interesse docente pela temática. Sabe-se que este profissional pode apresentar resistência pela implantação do novo, seja pela suposta inversão de papéis com o discente, seja pela falta de familiaridade com a temática e as tecnologias digitais, ou até mesmo pelo conformismo de ter sido ensinado por meio de técnicas tradicionais. Independente da causa, estudos como este podem suscitar o entendimento da necessidade de ruptura de paradigmas.

Ensaios como este permitem a reflexão crítica da prática docente e a incorporação de novas práticas e tendências educacionais capazes de melhorar o processo de ensinoaprendizagem. Parte-se do princípio que mais importante do que ser professor é o se refazer a todo tempo em sua profissão e acompanhar o ritmo acelerado das atualizações.

O presente artigo considera o processo educativo como um processo histórico, que se articula com a sociedade, sua multiplicidade de sujeitos, espaços e tempos. Nesse sentido, uma breve contextualização é realizada, partindo das metodologias tradicionais até as metodologias ativas.

Realiza-se um estudo de abordagem qualitativa como preconiza Pereira et al. (2018) por meio de uma revisão bibliográfica sobre a temática analisada.

Serão apresentadas em seguida boas práticas relacionadas à aprendizagem baseada em problemas, ensino híbrido e sala de aula invertida. Este estudo tem como objetivo realizar uma breve revisão sobre a temática e apresentar boas práticas de algumas metodologias de ensino-aprendizagem exitosas no âmbito do ensino superior.

\section{Metodologias de Ensino Tradicionais e Contemporâneas}

As metodologias de ensino são essenciais na resposta às novas demandas educacionais da sociedade capitalista. Essas estratégias apontadas pelo professor para serem utilizadas no processo de ensino-aprendizagem são escolhidas com base em experiências e evidências de desenvolvimento de habilidades pelos alunos (Beraza, 2011).

Os métodos tradicionais de ensino possuem características bem específicas, tais como: enfoque na memorização de informações, uso exclusivo da sala de aula tradicional e posição central do professor, que se apresenta como o detentor de todo o poder (Chan, 2013).

A sala de aula tradicional é considerada um subproduto do industrialismo e, assim como este vem sendo substituído pela economia do conhecimento, as salas de aulas e metodologias necessitam de mudanças. Nesse sentido, a Organização das Nações Unidas para a Educação, a Ciência e a Cultura (2009) e o documento Parâmetros Curriculares Nacionais 
(Brasil, 1988) propõem o incentivo à colaboração e investigação (Valente, 2014).

Para alcançar a mudança necessária no campo educacional do ensino superior, um dos primeiros conceitos a serem revistos é o de sala de aula universitária. Dessa forma, Masetto (2012) discute este conceito e atribui a ele o espaço e tempo no qual os atores de um processo de aprendizagem se unem para discutir, interagir e agir sobre determinados conteúdos. A partir daí, há o entendimento dos futuros locais de trabalho dos estudantes como locais onde a aprendizagem se aproxima mais significativamente da realidade.

Parte-se do princípio de que a vida intelectual é impossível sem abstrações e simbolizações e o uso de métodos que favoreçam estes processos é determinante para o sucesso do aprendizado. Métodos visuais, auditivos, cinestésicos, práticos e de leitura devem se complementar de forma a envolver o aluno no processo cognitivo.

É importante destacar que as diretrizes internacionais e nacionais para a educação superior apontam a necessidade de uma mudança no estilo tradicional de ensino, passando este a ser centrado no aluno e não na transmissão automática de conhecimentos. A ênfase é dada à formação por competências, que abrange um conjunto de conhecimentos, habilidades e atitudes e sua real aplicação na prática profissional, a partir de uma formação humanística (Cotta, et al., 2015).

Atualmente, um dos grandes problemas das instituições de ensino refere-se à sua rigidez institucional e consideráveis dificuldades em lidar com modificações em suas dinâmicas de funcionamento. $\mathrm{O}$ professor assume importante papel nos processos de mudança e o treinamento dos mesmos é essencial para a compreensão das transformações metodológicas, de acordo com as transições da sociedade e consequentes alterações de demandas dos "alunos digitais" (Callaham \& Dopico, 2016).

A literatura brasileira, em sua grande parte, considera as metodologias ativas como estratégias didáticas nas quais a centralidade do processo de ensino - aprendizagem é baseada no aluno, em contradição ao que é classificado como metodologia tradicional, que centra o processo no professor. Pode-se afirmar, no entanto, que as metodologias ativas são as estratégias que criam oportunidades de ensino por meio das quais o aluno pode ter um comportamento mais ativo e engajado com a aprendizagem (Valente, 2017).

Os diferentes tipos de metodologia compreendem quatro elementos: a forma como a informação é entregue; a organização espacial e temporal; o foco e gestão das atividades de aprendizagem e as relações interpessoais. A partir desses quatro elementos são estruturados alguns métodos: ensino baseado em casos, aprendizagem baseada em problemas, aprendizagem baseada em projetos e contratos cooperativos de aprendizagem (Beraza, 2011). 
Uma tendência natural de alguns docentes é replicar técnicas em que já esteve inserido. Análise de Dickson \& Ladefoged (2017) apontou que professores do ensino superior eram formados principalmente na área técnica e não na didática. Como reflexo de tal formação, a abordagem de ensino era centrada no poderio e centralização docente. Esses apresentavam resistência ao incorporar as metodologias de aprendizagem ativa.

Dentre os temores relatados pelos docentes, o que predomina é o de não conseguir completar o currículo. Tal situação leva o docente a questionar se os alunos aprendem tanto nas metodologias ativas quanto com os métodos tradicionais (Tabari, 2014).

Diversos autores trataram a questão da metodologia ativa, não chegando a um conceito comum, mas apresentando características que se complementam. Collins e O’Brien (2011) apontam que neste tipo de aprendizagem os alunos refletem sobre ideias e sua aplicação no mundo real, ou seja, são envolvidos em atividades de resolução de problemas, síntese de informação e aplicação. Para Prince (2004), tal metodologia abrange estratégias de aprendizagem colaborativa e baseada em problemas.

\section{Boas Práticas das Metodologias Ativas no Ensino Superior}

\subsection{Aprendizagem Baseada em Problemas}

A Aprendizagem Baseada em Problemas (ABP), também conhecida pela sigla PBL (Problem - Based Learning), foi aplicada inicialmente na década de 1950 na Escola de Medicina da Case Western Reserve University nos Estados Unidos e posteriormente na década de 1960 na Faculdade de Medicina da Universidade McMaster no Canadá. Tal metodologia visa aproximar o aluno de graduação à prática profissional que irá exercer (Gomes, et al., 2009).

O programa desta universidade canadense serviu de modelo para outros programas e baseou-se nos seguintes princípios: aprendizagem centrada no aluno e ocorrendo em pequenos grupos; professores como tutores ou mediadores da aprendizagem; utilização dos problemas como estímulo à aprendizagem; desenvolvimento das habilidades embasadas nos problemas e novas informações adquiridas a partir da aprendizagem autodirigida (Barrows, 1996).

A metodologia ABP foi criada em um curso de saúde e atualmente é utilizada nos mais diversos cursos de formação profissional. De acordo com Mello et al. (2015), 86,6\% dos estudos realizados com a ABP em cursos de graduação em saúde apresentam eficácia. 
Roca et al. (2016) compararam resultados de três metodologias de ensino (ABP, ensino baseado em casos e metodologias tradicionais) com 74 estudantes de Enfermagem em uma universidade na Espanha. O estudo utilizou um teste de desempenho para avaliar resultados de aprendizagem, pautando-se na Taxonomia de Bloom.

Os resultados apontam que os estudantes possuem maior experiência com a metodologia tradicional, porém, ao utilizar a ABP e a metodologia baseada em casos, os estudantes desenvolvem raciocínio de ordem superior, sendo capazes não apenas de aplicar o conhecimento, mas de analisar, avaliar e criar novas etapas.

Stentoft (2017) aborda três barreiras à abordagem ABP: a organização curricular, o desenvolvimento dos alunos para trabalhar com as questões interdisciplinares e o desenvolvimento das competências dos docentes para atender estas necessidades.

Em pesquisa realizada por Hemker et al. (2017) acerca da abordagem ABP utilizada em quatro seminários sobre avaliação educacional, algumas sugestões de melhoria ao método foram descritas, tais como: aprimoramento do conhecimento metodológico da ABP e seus objetivos, utilização de problemas de contextos múltiplos e adaptação ao conhecimento prévio flexível.

Ao afirmar que os métodos de trabalho dos pesquisadores matemáticos se assemelham a ABP e que o conceito de sociedade inclui estes pesquisadores, Dahl (2018) defende a ideia que os estudantes de engenharia precisam entender o mundo matemático e, para isso, descreve dois casos de aplicação da metodologia. No primeiro caso (matemática aplicada) foi solicitado um mecanismo de otimização de uma rota de coleta de ovos de agricultores na Dinamarca. Para a resolução do problema, os alunos precisavam se concentrar em ferramentas matemáticas existentes e otimizá-las, o que facilitou a discussão e formulação de soluções a partir da construção de um relatório.

No segundo caso (matemática pura) foi solicitada a solução de um problema de fluxo em uma rede localizada em território desconhecido. Por meio das duas problematizações o autor evidenciou o sucesso da ABP no ensino da engenharia e que embora a matemática em algumas situações possa requerer um conhecimento abstrato, a metodologia ABP pode ser uma importante ferramenta.

Feehily (2018) analisou a integração da ABP em um módulo sobre Resolução Internacional de Disputas Comerciais em um curso de direito envolvendo 28 alunos. Os resultados também foram favoráveis à metodologia: $85,71 \%$ dos alunos gostaram mais da ABP em comparação às aulas tradicionais; $71,43 \%$ consideraram esta metodologia mais realista e atrativa; além disso, 75\% dos envolvidos acreditavam que a ABP deveria ser 
obrigatória no ensino jurídico.

Em uma pesquisa online, 628 estudantes de uma universidade alemã foram contatados via e-mail e convidados a responder um questionário sobre os efeitos percebidos da $\mathrm{ABP}$ em um curso. Desse total, 150 responderam completamente o questionário de forma a compor a análise. De acordo com os resultados da pesquisa de Rossano et al. (2016), as principais motivações e benefícios relatados pelos participantes foram a aquisição de experiência prática em situações da vida real e o desenvolvimento de conceitos concretos.

Forcael et al. (2015) investigaram a aplicação da ABP em um curso de Engenharia Civil no Chile, comparando este método com a metodologia tradicional de ensino. Os alunos foram divididos em dois grupos e foram submetidos ao ensino pela $\mathrm{ABP}$ e por aulas expositivas. Após responderem a questões abertas, ficou evidenciada a eficiência da ABP no ensino e o alto grau de aceitação desta metodologia pelos estudantes de engenharia, que puderam resolver situações práticas e reais de sua futura profissão.

No campo da Enfermagem, Carbogim et al. (2017) realizaram uma meta-análise com estudos nacionais e internacionais sobre a utilização da ABP e concluíram que a literatura tem apontado positivamente para a mesma. Os autores enfatizaram a necessidade de maiores pesquisas do tipo intervenção com tal método e que dois fatores principais devem ser levados em consideração: a motivação do aluno e o conhecimento prévio que permite a assimilação de novos conhecimentos.

Várias experiências foram descritas na literatura, porém foram identificadas a fragmentação e fragilidade da maioria dos estudos, uma vez que tratam de disciplinas específicas ou momentos pontuais na formação profissional.

O grande desafio refere-se à continuidade da abordagem e para este desenvolvimento é necessário considerar a questão da capacitação docente. É fundamental proporcionar aos docentes, além das condições de trabalho (recursos e tempo de planejamento), o apoio necessário para as atualizações profissionais.

\subsection{Ensino Híbrido}

A inserção das Tecnologias da Informação e Comunicação (TIC's) no campo educacional é fundamental na sociedade contemporânea, que se encontra em uma era totalmente digital. As mudanças na sociedade demandam inovações nas práticas educacionais e nesse sentido, diversos autores têm tratado da temática, às vezes apenas com nomenclaturas diferentes: ensino híbrido (Valente, 2014); aprendizagem ubíqua (Santaella, 2014); sociedade 
onlife (Floridi, 2015).

As tecnologias digitais no processo de ensino estão relacionadas à necessidade de preparar adequadamente estudantes para o trabalho futuro e a aprendizagem ao longo da vida na sociedade (Chen, et al., 2014).

Até a década de 1980 os materiais educativos eram totalmente impressos e a partir desse período, as TIC's trouxeram mudanças significativas no cenário educacional. A partir de sua expansão foram criadas diferentes modalidades de ensino a distância, dentre elas, o ensino híbrido, que combina atividades presenciais e a distância (Valente, 2014).

No âmbito da educação formal, o ensino híbrido alterna o estudo online e momentos de ensino presencial com interação com o professor e demais alunos (Staker \& Horn, 2012).

Bralić \& Divjak (2018) pesquisaram um modelo de aprendizagem que combinava aula tradicional e Massive Open Online Courses (MOOC's) a partir de um diário de aprendizagem com perguntas abertas em um recurso da plataforma Moodle. O estudo envolveu alunos da Universidade de Zagreb matriculados no curso "Discrete Mathematics with Graph Theory", ofertados em programas de Sistemas de Informação e Engenharia de Software. O estudo avaliou os resultados em três anos e estes apontaram que os alunos que optaram pelo MOOC obtiveram notas finais mais altas. Porém, alguns alunos relataram a falta de conexão entre os conteúdos do MOOC e da sala de aula.

Com base nos demais resultados desta pesquisa, os autores destacaram algumas recomendações aos docentes: o fato de permitir que o aluno escolha o MOOC aumenta a sua motivação, ou seja, é necessário ofertar diversos tipos de cursos; a carga de atividades deve ser cuidadosamente analisada, pois a intenção é que os objetivos propostos sejam atingidos; e o retorno deve ser imediato.

Czepula et al. (2018) analisaram a implementação do ensino híbrido em duas disciplinas da Graduação em Farmácia na Universidade Federal do Paraná: Cuidados Farmacêuticos I e II. A pesquisa envolveu 133 alunos, que responderam a testes pré e pósinstrução idênticos, delineados a partir da revisão da Taxonomia de Bloom. Foram observadas diferenças significativas no pré e pós-teste e os alunos alcançaram níveis de domínio cognitivo elevados a partir da metodologia de ensino utilizada.

A implementação do ensino híbrido com ênfase nos alunos menos proficientes foi tema da pesquisa de Yusoff et al. (2017). Tal estudo teve como objetivo demonstrar a importância de identificar os estilos de aprendizagem dos alunos menos proficientes de forma a inserir novas tecnologias de ensino para favorecer o processo de ensino-aprendizagem. A pesquisa envolveu 64 estudantes em uma Universidade na Malásia e apontou o ensino híbrido 
como favorecedor das interações sociais e motivador acadêmico, melhorando o desempenho.

Um ponto importante enfatizado pelos autores é a importância do papel do professor. Eles apontam que a tecnologia é apenas uma ferramenta e cabe aos docentes seu uso exitoso.

No campo jurídico, Mercado (2016) realizou um estudo com o objetivo de aplicar e investigar o uso das TIC's como estratégia didática no ensino das leis. A proposta envolveu atividades que possibilitam aos docentes da área jurídica refletir a respeito da utilização de uma metodologia de ensino participativa, voltada para o envolvimento do aluno, levando o mesmo a vivenciar uma situação real, tornando esta vivência parte fundamental do processo de construção do aprendizado.

Ao analisar a transição de ambientes de aprendizagem tradicionais para as TIC's no ensino da graduação em Química, Barak (2007) apresentou o desenvolvimento de quatro ambientes de aprendizagem aprimorados pelas TIC's: página do curso, fórum, modelagem molecular computadorizada, e projetos. Os docentes instrutores concordaram na importância da incorporação das TIC's no ensino da Química e apontaram como principais barreiras a carga horária demandada para planejamento e a falta de conhecimentos e recursos para a efetivação da estratégia.

Um estudo desenvolvido por Wichadee (2015) apresentou um ensino híbrido que utiliza múltiplas ferramentas como ambiente de aprendizagem e apontou dados inerentes a aprendizagem, a participação, ao conforto com a tecnologia e a satisfação de 182 alunos matriculados em um curso de inglês.

A satisfação e o conforto apresentaram-se de forma moderada, porém a aprendizagem e participação tiveram índices satisfatórios. Estudos como este permitem a análise de benefícios e desvantagens do ensino híbrido em determinadas realidades, permitindo a reflexão docente acerca do processo metodológico adequado ao público.

É preciso considerar a existência de um "aluno digital" e, portanto, inseri-lo no seu contexto para que se sinta estimulado no processo formativo. Não há como negar a necessidade de um constante aprimoramento docente. É preciso compreender que nem todos os docentes estão familiarizados com as novas tecnologias, principalmente os que não pertencem à atual geração que "já nasce conectada". Dessa forma, um dos entraves à implantação de metodologias inovadoras, pautadas em novas tecnologias, tem sido a resistência de alguns docentes.

\subsection{Sala de Aula Invertida}


A Sala de Aula Invertida (SAI) foi proposta por Lage et al. (2000) e utilizada pela primeira vez em 1996 na Universidade de Miami. Após uma publicação sobre o assunto em 2010 no jornal "The New York Times", diversas experiências começaram a acontecer e serem publicadas (Pavanelo \& Lima, 2017).

De acordo com o relatório Flipped Classroom Field Guide (2014), existem algumas regras básicas para a execução da metodologia em questão: a) o momento em sala de aula envolve questionamentos, resolução de casos e problemas, e aplicação - ampliação do aprendizado online; b) retorno imediato; c) atividades presenciais e online valem nota; d) alto grau de planejamento nas atividades online e presenciais.

$\mathrm{Na}$ sala de aula invertida a instrução ocorre fora da sala de aula e antes da aula por meio de diversos recursos como vídeos, apresentações digitais, entre outros. O tempo em sala é destinado às discussões mais profundas sobre o tema, com a colaboração entre os alunos e o instrutor (Francl, 2014).

Sengel (2016) investigou os efeitos de videoaulas e atividades de casa em um curso de física, além de avaliar a percepção dos estudantes sobre o uso da SAI no ensino da física. Os resultados apontaram a eficácia do preparo prévio para a aula com o apoio de vídeos e atividades. Dessa forma, os alunos puderam ter um maior aproveitamento com as discussões em sala.

Resultados similares em outro curso de física foram observados por Aşıksoy \& Özdamlı (2016), que identificaram o aumento da realização e participação dos alunos ao utilizarem a aprendizagem ativa e adotarem o modelo de sala de aula invertida.

A alfabetização tecnológica influencia diretamente a prática docente. Um estudo realizado na University of North Dakota por Georgina e Olson (2008) apontou tal relação e revelou a importância do treinamento para a tecnologia nas universidades, objetivando o aprimoramento pedagógico.

Lopes e Soares (2018) apresentaram a experiência da SAI implementada em um curso de Matemática Financeira, investigando a aprendizagem e realização dos alunos. O estudo envolveu 803 alunos pesquisados durante os anos de 2014, 2015 e 2016. O impacto positivo foi identificado em quase todos os envolvidos.

No âmbito do ensino em saúde, Howard et al. (2017) descreveram o sucesso da implantação de três cursos no formato SAI em uma universidade americana. Todo o material (incluindo áudios, vídeos, artigos e livros) era disponibilizado aos alunos uma semana antes de cada aula. O tempo em aula foi utilizado principalmente em exercícios de aplicação no mundo real, estimulando formulação e debates acerca das estratégias de intervenção. Uma 
(CC BY 4.0) | ISSN 2525-3409 | DOI: http://dx.doi.org/10.33448/rsd-v9i7.3765

estratégia também utilizada foi o desenvolvimento da avaliação crítica dos trabalhos dos colegas, o que favoreceu o debate, interação e surgimento de novas ideias.

Porcaro et al. (2016) também obtiveram resultados positivos em seu estudo em um curso de Hematologia nesse formato. Foram identificadas melhorias no desempenho dos exames finais, que passaram de 47-48\% para 56-65\% a partir da aplicação da SAI.

Mclaughin et al. (2016) exploraram a implementação desta metodologia em duas instituições de ensino superior na Austrália e nos Estados Unidos. As duas instituições apontaram que para o sucesso da metodologia são necessárias abordagens sistemáticas e interativas na promoção da melhoria e aprimoramento contínuo. Esse processo de avaliação deve ser embasado no uso de melhores tecnologias e da estimulação da interação.

Na Universidade de King Abdulaziz na Arábia Saudita, Al-Zahrani (2015) investigou o impacto da SAI na promoção do pensamento crítico do aluno. A pesquisa foi desenvolvida no curso de Educação e dividiu os alunos em dois grupos, um utilizando a estratégia tradicional e o segundo utilizando a SAI. Como instrumento de coleta de dados foi utilizado um questionário que avaliou a percepção dos alunos. De acordo com a análise dos resultados, este método de ensino pode promover a criatividade no que se refere à fluência, flexibilidade e inovação, porém, a preparação dos alunos mostrou-se ainda limitada para tal.

Pavanelo \& Lima (2017) apresentaram os resultados da primeira experiência de SAI utilizada no Instituto Tecnológico de Aeronáutica (ITA), mais especificamente na disciplina de Cálculo Diferencial e Integral I. Os autores destacaram a necessidade de atitudes inovadoras no ensino de cálculo nos cursos superiores. $\mathrm{O}$ estudo apontou a dependência por parte dos alunos em relação à aula expositiva, destacando a importância da capacitação e motivação docente para o sucesso do método. Foram utilizados o endereço eletrônico da disciplina, videoaulas e leituras complementares, porém, houve necessidade de repensar alguns itens: tempo de videoaulas, didática docente no vídeo e enfoques de conteúdo. Os autores consideraram o estudo positivo e alternativa interessante para o aprimoramento da disciplina de cálculo.

No campo da matemática, Lo et al. (2017) realizaram uma revisão sistemática usando uma abordagem meta-analítica e apontaram que diversos docentes de matemática ainda não possuem conhecimento ou experiência para usar a SAI, sendo necessária uma maior proximidade com a temática.

Uma revisão sistemática sobre a SAI foi realizada por Betihalvas et al. (2016) envolvendo cinco estudos e 934 estudantes de Enfermagem de nível superior. De acordo com os autores, o ensino centrado no aluno oferece flexibilidade e potencialidade para o 
desenvolvimento do pensamento crítico. O uso da SAI vem aumentando no campo da enfermagem, porém, há necessidade de mais pesquisas referentes aos processos de implantação, experiências exitosas e evidências adicionais acerca do método.

Thai et al. (2017) examinaram a metodologia SAI em comparação com uma aprendizagem mista, uma aprendizagem tradicional e uma configuração de aprendizagem virtual em estudantes matriculados no segundo ano de um curso de graduação no Vietnã. Os resultados apontaram a superioridade dos resultados relacionados à SAI, uma vez que fornece maior tempo para processamento de informações e melhores oportunidades de envolvimento no processo ensino-aprendizagem.

Hu et al. (2018) analisaram a eficácia das salas de aula invertidas na formação de enfermeiros na China. Ao realizarem uma meta-análise de 11 trabalhos, puderam identificar a melhoria do conhecimento teórico e das habilidades profissionais. Os autores demonstraram a baixa qualidade metodológica dos trabalhos publicados com experiências sobre o assunto naquele país.

A SAI é uma forte tendência metodológica, porém, requer muito mais tempo de planejamento por parte docente e institucional, tornando-se mais dispendiosa se comparada à metodologia tradicional. Infelizmente, estudos que analisam o impacto dessas metodologias em longo prazo ainda são incipientes, o que impacta na realização de abordagens pontuais dentro de um currículo.

\section{Considerações Finais}

As mudanças e avanços na sociedade impactam diretamente as demandas educacionais e a universidade deve estar preparada para as alterações necessárias no que se refere aos processos formativos dos alunos e, consequentemente, nas metodologias de ensino utilizadas.

A partir das recomendações atuais (internacionais e nacionais) de um ensino centrado no aluno e de um maior protagonismo em seu processo cognitivo, muitos desafios surgem para os diferentes atores: instituições de ensino, gestores, docentes e discentes. Dentre os principais desafios destacam-se a falta de capacitação docente, a resistência aos novos formatos de ensino outrora desconhecidos, a falta de investimentos por parte das instituições, além da falta de motivação docente e discente.

As metodologias ativas devem estar cada vez mais presentes nos currículos do ensino superior. Este trabalho evidenciou aspectos promissores da aprendizagem baseada em problemas, ensino híbrido e da sala de aula invertida. Observa-se que as experiências com tais 
(CC BY 4.0) | ISSN 2525-3409 | DOI: http://dx.doi.org/10.33448/rsd-v9i7.3765

metodologias ainda são bem pontuais, necessitando de maior difusão no currículo. Embora exitosas, estas ainda aparecem de forma discreta na academia.

Embora o protagonismo das metodologias ativas seja do aluno, o planejamento e desenvolvimento são responsabilidade docente. Compete ao professor buscar novas tecnologias para o ensino e metodologias inovadoras. Este deve estar sempre se atualizando por meio de cursos, congressos, encontros e demais eventos que divulguem informações na área. A implantação e manejo de novas metodologias de ensino requer estudo prévio e é importante que o docente experimente uma de cada vez, detectando qual a de melhor adesão à sua realidade.

A implantação de metodologias de ensino mais ativas impacta diretamente a atração dos alunos, elevando os índices de aprendizagem e diminuindo a evasão escolar a partir de um processo cognitivo significativo.

Mais especificamente no ensino superior, as demandas por profissionais que solucionem situações complexas e sejam ativos em seus campos de atuação é uma realidade que reflete diretamente no trabalho docente. Sim! O docente direciona. Ele contribui com a formação de excelentes ou péssimos profissionais.

Paulo Freire (1981) concebia a ideia de que o ensino deve partir da realidade do aluno e o ponto-chave desta reflexão baseia-se nessa premissa. A realidade atual do aluno é uma "realidade digital" e por isso, é necessário incluir no processo de ensino-aprendizagem estas novas tecnologias e métodos.

O profissional que deseja atuar como professor deve ser sempre aberto ao novo, explorar novas possibilidades e se atualizar constantemente. Aprendizagem baseada em casos, em problemas, simulações, sala de aula invertida, ensino híbrido, mudança no conceito de sala de aula universitária, atividades online e práticas. Essas ideias devem permear a atuação de todo mediador de conhecimento que almeja o sucesso na aprendizagem de seu aluno.

\section{Referências}


Al-Zahrani, AM. (2015). From passive to active: The impact of the flipped classroom through social learning platforms on higher education students' creative thinking. British Journal of Educational Technology, 46(6), 1133-1148. doi: 10.1111/bjet.12353

Aşıksoy, G, \& Özdaml1, F. (2016). Flipped Classroom adapted to the ARCS Model of Motivation and applied to a Physics Course. Eurasia Journal of Mathematics, Science \& Technology Education, 12(6). doi: 10.12973/eurasia.2016.1251a

Barak, M. (2007). Transition from traditional to ICT-enhanced learning environments in undergraduate chemistry courses. Computers \& Education, 48(1), 30-43. doi: 10.1016/j.compedu.2004.11.004

Barrows, HS. (1996). Problem-based learning in medicine and beyond: A brief overview. New directions for teaching and learning, 1996(68), 3-12. doi: $10.1002 / \mathrm{t} l .37219966804$

Beraza, MAZ. (2011). Metodología docente. REDU: Revista de Docencia Universitaria, 9(3), 75.doi: 10.4995/redu.2011.6150

Betihavas, V., Bridgman, H., Kornhaber, R., \& Cross, M. (2016). The evidence for 'flipping out': A systematic review of the flipped classroom in nursing education. Nurse education today, 38, 15-21.doi: 10.1016/j.nedt.2015.12.010

Bralić, A \& Divjak, B. (2018). Integrating MOOCs in traditionally taught courses: achieving learning outcomes with blended learning. International Journal of Educational Technology in Higher Education, 15(1), 2.doi: 10.1186/s41239-017-0085-7

Callahan, BE \& Dopico, E. (2016). Science teaching in science education. Cultural Studies of Science Education, 11(2), 411-418.. doi: 10.1007/s11422-015-9703-7

Chan, ZC. (2013). A systematic review of critical thinking in nursing education. Nurse Education Today, 33(3): 236-240. doi: 10.1016/j.nedt.2013.01.007 
Chen, Y, Wang, Y \& Chen, NS. (2014). Is FLIP enough? Or should we use the FLIPPED model instead? Computers \& Education, 79, 16-27. doi: 10.1016/j.compedu.2014.07.004

Collins, JW \& O'Brien, NP. (2011). The Greenwood dictionary of education. ABC-CLIO. Carbogim, FDC., Oliveira, LBD, Mendonça, ÉTD, Marques, DA, Friedrich, DBDC \& Püschel, VADA. (2017). Ensino das habilidades do pensamento crítico por meio de problem based learning. Texto \& Contexto-Enfermagem, 26(4).doi: 10.1590/010407072017001180017

Dahl, B. (2018). What is the problem in problem-based learning in higher education mathematics. European Journal of Engineering Education, 43(1), 112-125.doi: 10.1080/03043797.2017.1320354

Dickson, M \& Ladefoged, SE. (2017). Introducing active learning pedagogy into a technical and vocational education and training academy in Kurdistan, Iraq. International Review of Education, 63(5), 679-702. doi: 10.1007/s11159-017-9660-3

Czepula, AIDS, Bottacin, WE, Júnior, EH, Pontarolo, R \& Correr, CJ. (2018). Active methodology and blended learning: An experience in pharmaceutical care. Currents in Pharmacy Teaching and Learning, 10(1), 106-111.doi: 10.1016/j.cptl.2017.09.013

Feehily, R. (2018). Problem-based learning and international commercial dispute resolution in the Indian Ocean. The Law Teacher, 52(1), 17-37.doi: 10.1080/03069400.2016.1273457 Flipped classroom field guide. Portal Flipped Classroom Field Guide.(2014). Disponível em: https://tlc.uic.edu/files/2016/02/Flipped-Classroom-Field-Guide.pdf.

Floridi, L. (Ed.). Onlife manifesto: being human in a hyperconnected era. London: Springer, 2015. Disponível em: <http://www.springer.com/us/book/9783319040929>.

Forcael, E, González, V, Orozco, F, Opazo, A, Suazo, Á \& Aránguiz, P. (2015). Application of problem-based learning to teaching the critical path method. Journal of Professional Issues in Engineering Education and Practice, 141(3), 04014016.doi: 10.1061/(ASCE)EI.19435541.0000236 
Francl, TJ. (2014). Is flipped learning appropriate?. Journal of Research in Innovative Teaching, 7(1). Disponível em: https://assets.nu.edu/assets/resources/pageResources/journalof-research-in-innovative-teaching-volume-7.pdf\#page $=128$.

Freire, P. (1981) Pedagogia do Oprimido. 9. ed. Rio de Janeiro: Paz e Terra.

Georgina, DA \& Olson, MR. (2008). Integration of technology in higher education: A review of faculty self-perceptions. The Internet and Higher Education, 11(1), 1-8.doi:

10.1016/j.iheduc.2007.11.002

Gomes, R, Brino, RDF, Aquilante, AG \& Avó, LRDSD. (2009). Aprendizagem Baseada em Problemas na formação médica e o currículo tradicional de Medicina: uma revisão bibliográfica. Revista brasileira de educação médica, 33(3), 433-40. doi: 10.1590/S010055022009000300014

Hemker, L, Prescher, C \& Narciss, S. (2017). Design and Evaluation of a Problem-Based Learning Environment for Teacher Training. Interdisciplinary Journal of Problem-Based Learning, 11(2), 10.Disponível em:

https://docs.lib.purdue.edu/cgi/viewcontent.cgi?article=1676\&context=ijpbl.

Howard, SW, Scharff, DP \& Loux, TM. (2017). Flipping classrooms in a School of Public Health. Frontiers in public health, 5, 73. doi: 10.3389/fpubh.2017.00073

Hu, R, Gao, H, Ye, Y, Ni, Z, Jiang, N \& Jiang, X. (2018). Effectiveness of flipped classrooms in Chinese baccalaureate nursing education: A meta-analysis of randomized controlled trials. International Journal of nursing studies, 79, 94-103.doi: 10.1016/j.ijnurstu.2017.11.01

Lage, MJ, Platt, GJ \& Treglia, M. (2000). Inverting the classroom: A gateway to creating an inclusive learning environment. The Journal of Economic Education, 31(1), 30-43.doi: $10.2307 / 1183338$

Lo, CK, Hew, KF \& Chen, G. (2017). Toward a set of design principles for mathematics flipped classrooms: A synthesis of research in mathematics education. Educational Research Review, 22, 50-73. doi: 10.1016/j.edurev.2017.08.002 
Lopes, AP \& Soares, F. (2018). Perception and performance in a flipped Financial Mathematics classroom. The International Journal of Management Education, 16(1), 105113.doi: 10.1016/j.ijme.2018.01.001

Masetto, MT. (2012). Competência pedagógica do professor universitário. Summus editorial. Disponível em: http://www.ucs.br/etc/revistas/index.php/conjectura/article/view/1267/891.

McLaughlin, JE, White, PJ, Khanova, J \& Yuriev, E. (2016). Flipped classroom implementation: a case report of two higher education institutions in the United States and Australia. Computers in the Schools, 33(1), 24-37.doi: 10.1080/07380569.2016.1137734

Mello, CDCB, Alves, RO \& Lemos, SMA. (2014). Methods of health education and training: literature review. Revista CEFAC, 16(6).doi: doi.org/10.1590/1982-0216201416012

Mercado, LPL. (2016). Metodologias de ensino com tecnologias da informação e comunicação no ensino jurídico. Avaliação: Revista da Avaliação da Educação Superior, 21(1), 263-99. doi: 10.1590/S1414-40772016000100013

Cotta, RMM, Costa, GDD \& Mendonça, ETD. (2015). Portfólios crítico-reflexivos: uma proposta pedagógica centrada nas competências cognitivas e metacognitivas. InterfaceComunicação, Saúde, Educação, 19, 573-588. doi: 10.1590/1807-57622014.0399.

Pavanelo, E \& Lima, R. (2017). Sala de Aula Invertida: a análise de uma experiência na disciplina de Cálculo I. Bolema: Boletim de Educação Matemática, 31(58), 739-759. doi: 10.1590/1980-4415v31n58a11.

Pereira, AS et al. (2018). Metodologia da pesquisa científica. [e-book]. Santa Maria. Ed. UAB/NTE/UFSM. Acesso em: 16 maio 2020. Disponível em: https://repositorio.ufsm.br/bitstream/handle/1/15824/Lic_Computacao_MetodologiaPesquisa-Cientifica.pdf?sequence $=1$.

Porcaro, PA, Jackson, DE, McLaughlin, PM \& O’Malley, CJ. (2016). Curriculum design of a flipped classroom to enhance haematology learning. Journal of Science Education and Technology, 25(3), 345-357.doi: 10.1007/s10956-015-9599-8 
Prince, M. (2004). Does active learning work? A review of the research. Journal of engineering education, 93(3), 223-231.Disponível em: https://www.engr.ncsu.edu/wpcontent/uploads/drive/1smSpn4AiHSh8z7a0MHDBwhb_JhcoLQmI/2004-Prince_AL.pdf.

Roca, J, Reguant, M \& Canet, O. (2016). Learning outcomes of “The Oncology Patient” study among nursing students: A comparison of teaching strategies. Nurse education today, 46, 2935.doi: 10.1016/j.nedt.2016.08.018

Rossano, S, Meerman, A, Kesting, T \& Baken, T. (2016). The Relevance of Problem-based Learning for Policy Development in University-Business Cooperation. European Journal of Education, 51(1), 40-55.doi: 10.1111/ejed.12165

Santaella, L. (2014). A aprendizagem ubíqua na educação aberta. Revista Tempos e Espaços em Educação, 15-22. doi: 10.20952/revtee.v0i0.3446

Şengel, E. (2016). To FLIP or not to FLIP: Comparative case study in higher education in Turkey. Computers in Human Behavior, 64, 547-555. doi: 10.1016/j.chb.2016.07.034

Staker, H \& Horn, MB. Classifying K-12 blended learning. Mountain View, CA: Innosight Institute, Inc. (2012). Disponível em: <http://www.christenseninstitute.org/wpcontent/uploads/2013/04/Classifying-K-12-blended-learning.pdf>.

Stentoft, D. (2017). From saying to doing interdisciplinary learning: Is problem-based learning the answer?. Active Learning in Higher Education, 18(1), 51-61.doi: $10.1177 / 1469787417693510$

Tabari, R. (2014). Education reform in the UAE: An investigation of teachers' views of change and factors impeding reforms in Ras Al Khaimah schools. Ras Al Khaima, UAE. doi: 10.18502/aqf.0112

Thai, NTT, Wever, B \& Valcke, M. (2017). The impact of a flipped classroom design on learning performance in higher education: Looking for the best "blend" of lectures and guiding questions with feedback. Computers \& Education, 107, 113-126.doi:

10.1016/j.compedu.2017.01.003 
Valente, JA. (2014). Blended learning e as mudanças no ensino superior: a proposta da sala de aula invertida. Educar em Revista, (4), 79-97. doi: 10.1590/0104-4060.38645.

Valente, JA, Almeida, MEB \& Geraldini, AFS. (2017). Metodologias ativas: das concepções às práticas em distintos níveis de ensino. Revista Diálogo Educacional, 17(52), 455-478. doi: 10.7213/1981-416X.17.052.DS07

Wichadee, S. (2015). Factors related to students' performance of hybrid learning in an English language course. In Curriculum Design and Classroom Management: Concepts, Methodologies, Tools, and Applications (pp. 225-241). IGI Global.

Yusoff, S, Yusoff, R \& Md Noh, NH. (2017). Blended learning approach for less proficient students. SAGE Open, 7(3), 2158244017723051. doi: 10.1177/2158244017723051.

\section{Porcentagem de contribuição de cada autor no manuscrito}

Camila Henriques Nunes $-55 \%$

Mario Sergio Michaliszyn - 45\% 